\title{
Alternativa para determinação de altitudes normais-ortométricas na Amazônia Legal Brasileira
}

\author{
Eurico Lourenço Nicacio Junior*
}

Recebido 20 de junho de 2018; aceito 14 de outubro de 2018

\begin{abstract}
The present paper intends to develop an alternative methodology for prediction of normal-orthometric heights through Brazilian Legal Amazon Rain Forest, a region that imposes logistical-operational difficulties for fieldwork and in which there is considerable precariousness in the Brazilian Fundamental Altimetric Network (BFAN), evidencing the need to improve the network in question to fully align with the current recommendations for the achievement of the International Height Reference Frame. An alternative strategy is based on GNSS observations and the use of global geopotential models (GGMs); alternatively, it uses the national geoid model MAPGEO2015 to evaluate employability. To do so, an approach in three different variants is performed: a) use of absolute and relative approaches for altimetric determination; b) use of GGM extracts obtained in different degrees of development and MAPGEO2015; and c) the use of geoid height and height anomaly for normal-geoid height modeling. Data regarding GNSS observations are extracted from the stations of the SAT-GPS network, coinciding with BFAN stations, with information of both ellipsoidal and normal-orthometric heights; GGMs extracts are obtained pointwisely for the main current combined MGGs. Given the low mean accuracy of data in the study area and its low linkage with BFAN, it is chosen to adopt a test area - the state of Paraná - best suited for elaboration and solidification of the strategy for a later application in the area of study. From the studies conducted in the test area, there is an unquestionable advantage of using the relative approach over the absolute one allied to GGMs, with an average of $72 \%$ better results for extracts developed up to
\end{abstract}

* Setor de Ciências da Terra, Departamento de Geomática, Programa de Pós-Graduação em Ciências Geodésicas, Universidade Federal do Paraná, UFPR, R. Cel. Francisco Heráclito dos Santos, 210, Jardim das Américas, 81531-970, Curitiba, PR, Brasil, e-mail: euriconicaciojr@gmail.com. 
degrees 2190 and 720; MAPGEO2015 was an exception to such results, presenting no adequacy to the relative approach. For the other analyzed variables - different functional and GGMs - expected non-standardized behavior is achieved, with only MGG and functional signaling for best modeling the normal-geoid height in the area called optimal configuration. It is discussed a strategy for validating results and treating outliers. A consolidated strategy is then elaborated, which is applied to the study area. With this application, normal-orthometric heights of 602 points are calculated in the Brazilian Legal Amazon Rain Forest, based on the relative method and the optimal configuration for the region. After treatment of outliers, it is verified that this procedure produces an average discrepancy of $13.87 \mathrm{~cm}$ in a controllable area, unlike the one with the absolute method and the national geoid model, which produces average discrepancy of $36.28 \mathrm{~cm}$ for the same stations. As an additional contribution, an estimate is made for the offset between the Brazilian vertical Data based on the methodology elaborated, resulting in a vertical distance of approximately $1.32 \pm 0.07$ $\mathrm{m}$, a consistent result regarding other determinations already carried out.

Key words: normal-orthometric heights; Brazilian Legal Amazon Rain Forest; alternative methodology; GGMs; relative method.

\section{Resumo}

O presente trabalho tem por objetivo desenvolver uma metodologia alternativa para predição de altitudes normais-ortométricas ao longo da Amazônia Legal Brasileira, região que impõe dificuldades logístico-operacionais para empreitadas de campo e na qual há considerável precariedade na Rede Altimétrica Fundamental Brasileira (RAFB), evidenciando necessidade de melhoria da rede em questão para um pleno alinhamento às atuais recomendações e esforços para consecução de uma vindoura Rede Internacional de Referência Altimétrica. A estratégia alternativa baseia-se em observações GNSS e na utilização de modelos globais do geopotencial (MGGs); alternativamente, utiliza-se o modelo geoidal nacional MAPGEO2015 para avaliação de empregabilidade. Para tanto, realiza-se uma abordagem com comparação em três variantes distintas: a) utilização dos métodos absoluto e relativo para determinação altimétrica; b) utilização de extratos dos MGGs obtidos em diferentes graus de desenvolvimento e do MAPGEO2015; e c) utilização das funcionais altitude geoidal e anomalia de altitude para modelagem de altitude normal-geoidal. Dados referentes às observações GNSS são extraídos das estações da rede SAT-GPS com coincidência com estações da RAFB, dotadas de informação de altitudes elipsoidal e normal-ortométrica; extratos dos MGG são obtidos ponto-a-ponto para os principais MGGs combinados da atualidade. Tendo em vista a baixa precisão média de dados na área de estudo e sua baixa vinculação com a RAFB, opta-se por adotar uma área-teste — o estado do Paraná- melhor comportada para elaboração e solidificação da estratégia para uma posterior aplicação na área de estudo. Dos estudos realizados na área- 
teste, verifica-se inquestionável vantagem de utilização do método relativo sobre o absoluto aliado aos MGGs, sendo em média $72 \%$ melhores para extratos desenvolvidos até graus 2190 e 720; o MAPGEO2015 figurou como exceção aos resultados, não havendo adequação com a abordagem relativa. Para as outras variáveis analisadas - diferentes funcionais e MGGs_ — há esperado comportamento não padronizado, havendo apenas sinalização de MGG e funcional que melhor modelam a altitude normal-geoidal na área denominada configuração ótima. Discute-se, ainda, um estratégia para validação dos resultados e tratamento de outliers. Elabora-se, então, uma estratégia consolidada, a qual é aplicada à área de estudo. Com esta aplicação, são calculadas altitudes normais-ortométricas de 602 pontos na Amazônia Legal Brasileira, com base no método relativo e na configuração ótima para a região. Após tratamento de outliers, verifica-se que tal procedimento produz discrepância média de 13,87 cm em uma área controlável, diferentemente do ocorrido com o uso do método absoluto e o modelo geoidal nacional, o qual produz discrepância média de $36,28 \mathrm{~cm}$ para as mesmas estações. Como contribuição adicional, realiza-se uma estimativa para o offset entre os data verticais brasileiros com base na metodologia elaborada, auferindose afastamento de aproximadamente $1,32 \pm 0,07 \mathrm{~m}$, resultado deveras coerente com as demais determinações já realizadas.

Palavras-chave: altitudes normais-ortométricas; Amazônia Legal Brasileira; metodologia alternativa; MGGs; método relativo.

\section{Resumen}

El presente trabajo tiene por objetivo desarrollar una metodología alternativa para predicciones de altitudes normales-ortométricas a lo largo de la Amazonia Legal Brasileña, región que impone dificultades logísticas-operacionales para trabajos decampo y en la que hay considerable precariedad en la Red Altimétrica Fundamental Brasileña (RAFB), evidenciando necesidades de mejora de las redes en cuestión para una completa alineación a las actuales recomendaciones y esfuerzos para la consecución de una Red Internacional de Referencia Altimétrica. Una estrategia alternativa se basa en observaciones GNSS y en la utilización de modelos globales y geopotenciales (MGGs); alternativamente se utiliza el modelo geoidal nacional MAPGEO2015. Para ello, se utiliza un enfoque de comparación de tres variantes distintas: a) utilización de métodos absolutos o relativos para determinación altimétrica, b) utilización de extractos de los MGGs obtenidos en diferentes grados de desarrollo y del MAPGEO2015, y c) utilización de las funcionalidades altitud geoidal y anomalía de altitud para modelado de altitud normal-geoidal. Datos referentes a observaciones GNSS son extraídos de las estaciones de la rede SAT-GPS en coincidencia con estaciones RAFB, dotadas de información de altitudes elipsoidal y normalortométricas; los extractos de los MGG se obtienen punto a punto para los principales MGG combinados de la actualidad. En vistas de la baja precisión media de datos en 
el área de estudio y su baja vinculación con la RAFB, se adopta un área de prueba — el estado de Paraná- que se comporta mejor para la elaboración y consolidación de estrategias para aplicar posteriormente en el área de estudio. De los estudios realizados en el área de estudio, se verifica una incuestionable ventaja de utilización del método relativo sobre el absoluto aliado a los $\mathrm{MGG}$, siendo en promedio un $72 \%$ mejores para extractos desarrollados hasta grados 2190 y 720; o MAPGEO2015 como una excepción a los resultados, no habiendo adecuación con el enfoque relativo. Para otras variables analizadas - diferentes funciones y MGGs - se observó un comportamiento no estandarizado, habiendo a la vez una señal de MGG y funcional que mejor modela la altitud normal-geoidal en el área denominada configuración óptima. Se discute, además, una estrategia para la validación de los resultados y tratamiento de outliers, y se elaboró una estrategia consolidada que se aplica al área de estudio. Con esta aplicación, se calculan altitudes normales-ortométricas de 602 puntos en la Amazonia Legal Brasileña, con base en el método relativo y en la configuración óptima para la región. Después del tratamiento de outliers, se verifica que tal procedimiento produce discrepancia media de $13.87 \mathrm{~cm}$ en un área controlable, a diferencia de lo ocurrido con el uso del método absoluto y el modelo geoidal nacional, el cual produce discrepancia media de $36.28 \mathrm{~cm}$ para las mismas estaciones. Como contribución adicional, se realiza una estimación para el off-set entre los datos verticales brasileños con base en la metodología elaborada, obteniéndose alejamiento de aproximadamente $1.32 \pm 0.07 \mathrm{~m}$, resultado verdaderamente coeficiente con las demás determinaciones ya realizadas.

Palabras clave: altitudes normales-ortométricas; Amazonia Legal Brasileña; metodología alternativa; $M G G$; ; método relativo.

\section{Contextualização e Estado-da-arte}

Conforme já discorrido por renomados geodesistas, tais como Hofmann-Wellenhof e Moritz (2006), Torge e Müller (2012) e Gemael (2012), o objetivo primordial da Geodésia é determinar a forma e a dimensão da Terra, bem como do campo da gravidade em seu exterior e suas variações temporais. Entretanto, ao longo das últimas décadas este objetivo tem passado por drásticas mudanças, principalmente devido à evolução das técnicas aplicadas em observações e dos métodos computacionais empregados em seu processamento. Concomitantemente a estes avanços, os problemas clássicos da Geodésia foram expandidos, e sua função primária de medição da superfície terrestre foi ampliada para a detecção dos efeitos de mudanças globais e da geodinâmica (Drewes, 2006).

Este entendimento traz à tona a necessidade de compreensão de entes físicos ligados diretamente à maneira como a superfície terrestre se dispõe e se modifica ao 
longo do tempo, gerando uma compreensão de como o Sistema Terra se comporta e trazendo consigo a possibilidade de monitorá-lo de modo contínuo. No entanto, para que isto seja possível, não apenas as observações contínuas são necessárias, mas também sua vinculação a um Sistema Geodésico de Referência Global.

Quando se trata de informações altimétricas, conforme preconizado por IAG (2015), a busca pela definição de um Sistema Vertical de Referência Global (do inglês, International Height Reference System-IHRS) deve ser realizada tomando-se como referência uma superfície equipotencial do campo da gravidade, com valor de geopotencial $W_{0}=62.636 .853,4 \mathrm{~m}^{2} \mathrm{~s}^{-2}$. Adicionalmente, IAG (2015) determina que as coordenadas verticais primárias para tal sistema de referência sejam definidas com base no número geopotencial $C_{P}$, expresso conforme a Equação 1, na qual $W_{0}$ representa o valor do geopotencial na superfície de referência e $W_{P}$ representa o valor do geopotencial no ponto de cálculo.

$$
C_{P}=-\Delta W_{P}=W_{0}-W_{P}
$$

Estas considerações supramencionadas são possíveis, na atualidade, devido principalmente a avanços tecnológicos ocorridos nas últimas décadas. Graças ao surgimento de modernas técnicas de análise do campo gravitacional, aliado ao precípuo desenvolvimento analítico e matemático segundo formulação adequada, não há mais a dependência de observações de campo ou observações terrestres como fonte única de dados. Um exemplo desta modernização pode ser visualizado nas informações obtidas de missões espaciais, algumas das quais têm por objetivo determinar as perturbações nas órbitas dos satélites. Estas perturbações são induzidas pelo efeito gravitacional de heterogeneidades de massas relativamente a uma distribuição esférica homogênea, gerando conhecimento das anomalias associadas às feições de longos e médios comprimentos de onda deste campo, e completadas com informações de curtos comprimentos de outras fontes. Quando associadas ao potencial centrífugo, viabilizam o estabelecimento de Modelos Globais do Geopotencial-MGGs (Melo, 2011).

Os MGGs são gerados a partir de um conjunto de coeficientes do desenvolvimento do potencial gravitacional em série de funções harmônicas esféricas. Todo esse arcabouço de informações, por fim, propicia a realização de determinações para implantação do IHRS, ao qual as observações altimétricas devem estar vinculadas.

No entanto, tais modificações e avanços não vêm a depor totalmente contra os dados altimétricos já existentes em cada país. A integração entre estes dados já coletados pelos órgãos competentes, visando à adequação à nova modelagem vertical e a eliminação de discrepâncias entre os data verticais nacionais e o datum vertical global, pode ser realizada conforme a formulação apresentada pela Equação 2, como adaptado de Santacruz Jaramillo et al. (2016), expressa em função da diferença entre 
os números geopotenciais de um ponto de cálculo $P$ obtidos segundo o datum global $\left(\mathrm{C}_{\mathrm{P}}\right)$ e o datum nacional $\left(\mathrm{C}_{\mathrm{Pi}}\right)$.

$$
C_{P}-C_{P_{i}}=W_{0}-W_{0_{i}}=-\delta W_{i} \approx \overbrace{\left[W_{0}-\left(U_{P}+T_{P}\right)\right]}^{I}-\overbrace{\sum_{j} g_{m_{j}} \Delta n_{j}}^{I I}
$$

Note que o desenvolvimento da diferença de números geopotenciais à esquerda da Equação 2 culmina na diferença dos termos I e II. O termo I contém a diferença entre o geopotencial de referência $W_{0}$ e a soma do esferopotencial $U_{P}$ em um ponto de interesse $P$ e o potencial perturbador $T_{P}$ no mesmo ponto $P$ - o primeiro é padronizado, como já descrito neste mesmo item; para o segundo, há fácil equacionamento matemático; para o terceiro, busca-se alternativas na atualidade para sua determinação plena - por meio da solução do Problema de Valor de Contorno da Geodesia (PVCG) - em pontos da superfície. Já o termo II contém o somatório de $j$ produtos discretos entre valores médios da gravidade $g_{m}$ e desníveis $\Delta n$, característicos de conjugação de operações de nivelamento e gravimetria, formando a parte prática da equação. Dessa forma, considerando-se a existência de uma rede de nivelamento com densidade adequada e conjugada com informações de gravimetria ao longo de seu levantamento, é possível unir este conhecimento prático à modelagem matemática teórica cabível e proporcionar o alinhamento com parte das recomendações que buscam a definição do IHRS.

No que diz respeito à integração brasileira a esta tendência mundial, verifica-se carência na união entre prática e teoria. Atualmente, a Rede Altimétrica Fundamental Brasileira (RAFB) - denominada Rede Altimétrica de Alta Precisão (RAAP), mantida pelo Instituto Brasileiro de Geografia e Estatística (IBGE) e vinculada ao Sistema Geodésico Brasileiro (SGB) conta com aproximadamente 69.859 estações de altitudes normais-ortométricas (altitudes obtidas por nivelamento geométrico e corrigidas do não-paralelismo das superfícies equipotenciais do campo da gravidade) conhecidas, distribuídas ao longo do território nacional (IBGE, 2011). Todavia, a maneira nãouniforme com que se distribui, a inexistência de vínculo de parte da rede com o Datum Vertical Brasileiro em Imbituba (DVB-I) e a existência de grande quantidade de ramais, os quais impossibilitam o ajustamento das altitudes trazem à tona um problema maior do que a aparentemente simples conversão e adequação às recomendações do IHRS vindouro: as deficiências atuais da RAFB. É possível verificar que, na Região Amazônica, deve haver um esforço para preenchimento de informações altimétricas, visando a uma melhor solução para o alinhamento ao IHRS.

Surge, então, a perspectiva de modelagem das altitudes normais-ortométricas $H^{N O r t}$ nas regiões de vazio tomando por base altitudes elipsoidais $h$ - provenientes de 
observações GNSS (Global Navigation Satellite System) discretas - e valores de altitude normal-geoidal $\eta$ - provenientes de cálculo de funcionais de MGGs. Esta modelagem baseia-se na resolução da equação 3, descrita como método absoluto, e cujos termos já foram definidos para um dado ponto $P$ (Gemael, 2012).

$$
h_{P} \approx H_{P}^{\text {Nort }}+\eta_{P} \Leftrightarrow H_{P}^{\text {Nort }} \approx h_{P}-\eta_{P}
$$

Como o enfoque principal deste trabalho reside na determinação de altitudes normais-ortométricas, em consonância com a RAFB, uma outra observação deve ser feita a seu respeito e sobre o exposto na equação 3. Conforme abordado por Ferreira et al. (2013), o fato do comportamento das altitudes normais-ortométricas não apresentarem um significado completamente físico faz com que estas estejam referidas a uma superfície de referência diferente das usuais geoide e quasi-geoide. Em virtude disso, a referência supracitada descreve a dependência entre as altitudes elipsoidais e as altitudes normais-ortométricas aos moldes da Equação 3, na qual $\eta$ é por eles denominada como altitude normal-geoidal, em analogia à altitude normal-ortométrica.

Uma representação da equação 3 é apresentada na porção à esquerda da Figura 1, a qual descreve a relação entre as variáveis supramencionadas para o ponto $P$ em questão e considerando a melhor modelagem da altitude normal-geoidal $\eta$ pela própria altitude geoidal $N$.

Todavia, chama-se a atenção para o seguinte fato: tanto os MGGs que dão origem às funcionais utilizadas para modelar altitudes normais-geoidais desejadas, quanto outros modelos de ondulação geoidal, como o nacional MAPGEO2015, contêm erros inerentes a seu sistema de processamento e a seu modo de obtenção na forma: $\eta_{P}{ }^{\text {modelo }}=\eta_{P}{ }^{\text {real }}+\varepsilon \Rightarrow \varepsilon=\eta_{P}{ }^{\text {modelo }}-\eta_{P}{ }^{\text {real }}$, que variam segundo parâmetros de especificação do modelo (Barthelmes; Köhler, 2016). Para solucionar este problema, Arana (2000) e Featherstone (2002) propõe a utilização do método relativo ao invés do método absoluto, o qual se baseia na utilização de um ponto origem $P_{0}$ como referência, com altitude normal-ortométrica $H^{\text {NOrt }}$, altitude elipsoidal $h_{0}$ e altitude normal-geoidal $\eta_{0}$ conhecidas e pela abordagem matemática apresentada na Equação 4.

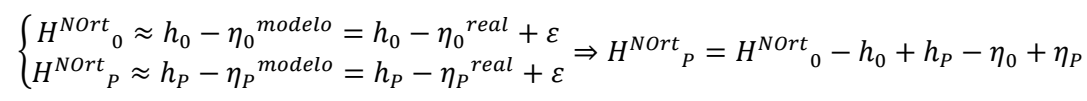

Ainda, a já mencionada Figura 1, quando analisada por completo, traz a representação da relação apresentada na Equação 4, novamente considerando a modelagem da altitude normal-geoidal $\eta$ pela altitude geoidal $N$. 


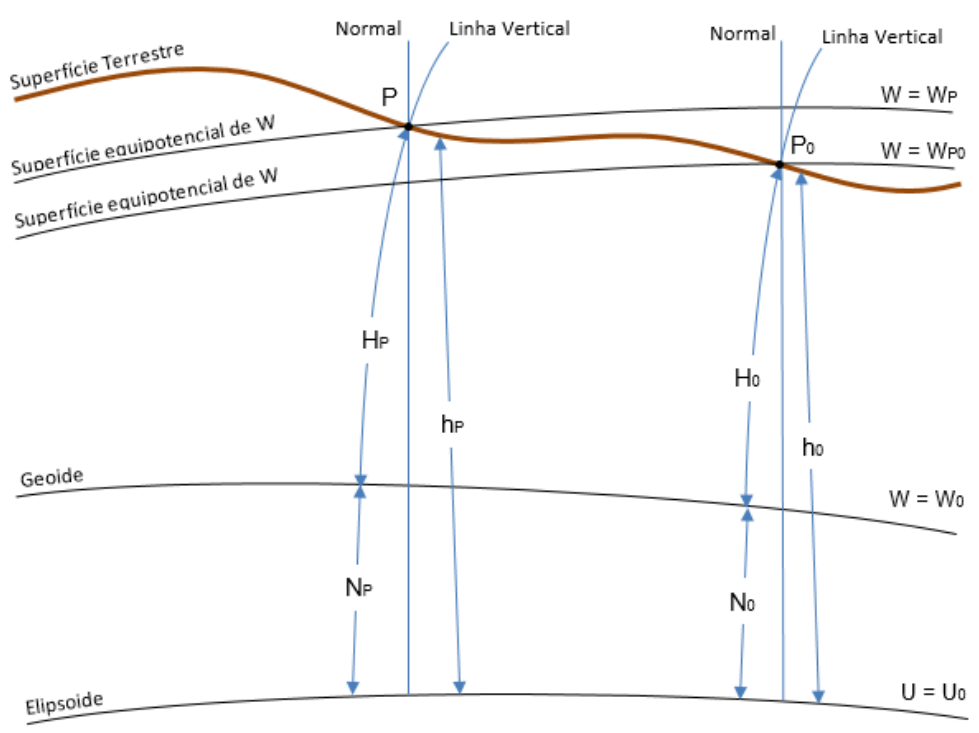

Figura 1. Modelagem altimétrica baseada em altitude elipsoidal e altitude geoidal. Fonte: Nicacio, 2017.

Por fim, frisa-se que a alternativa apresentada anteriormente é reforçada por Sánchez (2016), principalmente sob o aspecto de consecução de sistemas de referência vertical. Segundo adaptado desta referência, as atuais técnicas geodésicas, principalmente aquelas apoiadas por posicionamento e navegação satelital, apresentam requisitos de precisão superiores àqueles propiciados pela Equação 3. Sendo assim, a utilização do método relativo para correlacionar altitude elipsoidal e altitude normalortométrica com a altitude normal-geoidal, conforme reescrito na Equação 5, deve ser utilizado para fins de maior precisão e para a viabilização de modernos e precisos sistemas de referência vertical.

$$
H_{P}=H_{0}-h_{0}+h_{P}-\eta_{0}+\eta_{P} \Leftrightarrow \Delta H=\Delta h-\Delta \eta
$$

Desta forma, a abordagem de determinação de altitude normal-ortométrica pelo método relativo, com o uso de observações GNSS discretas e informações geoidais provenientes de Modelos Globais do Geopotencial, apresenta grande potencial de solução para a densificação da RAFB na região da Amazônia Legal Brasileira e, consequentemente, para viabilizar o alinhamento da rede ao IHRS e sua conveniente materialização. Justifica-se, portanto, a realização do presente trabalho, que visa a verificar a possibilidade e a viabilidade da referida abordagem para a área estudada. 


\section{Materiais e métodos}

\section{Dados altimétricos e GNSS}

Conforme discorrido por IBGE (2017), o SGB (Sistema Geodésico Brasileiro) é composto fundamentalmente pelas redes vertical, horizontal e gravimétrica. Com o advento das tecnologias espaciais de posicionamento, estas redes abrangem na atualidade todo o território nacional. No que diz respeito à rede planimétrica, uma de suas vertentes, na atualidade, é a rede geodésica composta por estações SAT-GPS, ou seja, estações cujas coordenadas foram determinadas por meio de rastreio e obtenção de informações da constelação GPS. Ainda segundo a referência citada, a rede SAT-GPS fornece um referencial com abrangência nacional para usuários que precisem de informações posicionais para diversos fins, como apoio ao mapeamento, demarcação de unidades político-administrativas, obras de engenharia, regulamentação fundiária, posicionamento de plataformas de prospecção de petróleo, delimitação de regiões de pesquisas geofísicas, entre outros. Além de informações planimétricas, as estações GNSS fornecem também coordenadas altimétricas referenciadas ao elipsoide - as altitudes elipsoidais.

Optou-se por realizar o acesso às informações geoespaciais por meio do Visualizador da INDE (Infraestrutura Nacional de Dados Espaciais, 2016) principalmente por ser possível realizar o download das referidas informações em formato shapefile. Esta característica possibilitou integração com ferramentas de geoprocessamento, como o software QGIS, a realização de operações espaciais com os dados, como recorte e interseção conforme necessário, e a fácil exportação das informações shapefile para outros formatos, em vistas de integrá-las a algoritmos desenvolvidos pelo autor.

Para a obtenção de dados da RAFB, também foi utilizado o Visualizador da INDE, com operação similar e visando a alcançar as vantagens já citadas. Um detalhamento sobre quantos e quais pontos foram utilizados no desenvolvimento da pesquisa é fornecido no item que discorre sobre área de estudo e área-teste.

\section{Modelos Globais do Geopotencial empregados e o Modelo Geoidal Nacional}

O primeiro modelo baseado não apenas na solução de estimativas de um conjunto de anomalias de gravidade provenientes de observações de órbitas satelitais, mas também provido de observações gravimétricas, observações sobre a topografia do terreno e de altimetria por satélite foi o EGM2008 (Pavlis et al., 2012). Este modelo foi considerado revolucionário à época de sua elaboração e lançamento, devido à grande quantidade de informações utilizadas para geração dos coeficientes de seu desenvolvimento em harmônicos esféricos e, consequentemente, de seu possível desenvolvimento até grau 2159, sendo completado em zonais até grau 2190. Levando em 
consideração esta mesma característica, foram selecionados para emprego no presente trabalho modelos combinados - ou seja, que integram informações gravitacionais derivadas de estudo de órbitas satelitais, informações de gravimetria terrestre e informações de altimetria por satélite - com possibilidades compatíveis com as do modelo EGM2008, principalmente no que diz respeito ao alto grau de desenvolvimento e à alta resolução espacial. A lista e as características dos modelos utilizados encontram-se na Tabela 1. Na dissertação, é realizada uma descrição detalhada sobre cada um dos modelos, a qual é omitida do presente artigo mas pode ser verificada em Nicacio e Dalazoana (2017).

Tabela 1

MGGs utilizados no trabalho

\begin{tabular}{crc}
\hline Modelo & Grau/Ordem empregados & Ano \\
\hline XGM2016 & 719 e 360 & 2017 \\
GOCO05C & 720 e 360 & 2016 \\
GGM05C & 360 & 2016 \\
EIGEN-6C4 & 2190,720 e 360 & 2014 \\
EGM2008 & 2190,720 & \\
e 360 & 2008 & \\
\hline
\end{tabular}

Fonte: $\quad$ adaptado de Nicacio (2017).

Reforça-se que, além das justificativas ora apresentadas, embasa-se esta escolha com resultados e desempenhos positivos para modelos combinados, em especial o próprio EGM2008, em estudos ao longo de todo o planeta, tais como Arábia Saudita (Al-Ghamdi; Dawod, 2014), Alemanha (Voigt; Denker, 2015), Polônia (Godah et al., 2015), América do Sul (Matos et al., 2015), África (Abd-Elmotaal, 2015) e, nacionalmente, na Amazônia oriental (Moreira, 2015).

Para a obtenção de extratos de funcionais dos MGGs a partir do serviço de cálculo no sítio do ICGEM (International Centre for Global Earth Models), organização responsável por armazenar, gerir e disponibilizar os modelos ao usuário, optou-se por uma abordagem ponto-a-ponto em detrimento de uma geração em grade e posterior interpolação, a fim de preservar a precisão e as características de cada modelo. Para tanto, foi desenvolvida e empregada a ferramenta SPGG - Single Point GEM [Global Earth Models] Generator, uma aplicação standalone capaz de gerar extratos dos modelos globais do geopotencial de forma pontual, utilizando uma lista de pontos elaborada e formatada pelo usuário e com a interação direta com o serviço web do ICGEM (Nicacio, 2016). 
Adicionalmente ao uso dos MGGs, utilizou-se para modelagem da altitude normal-geoidal o modelo geoidal nacional MAPGEO2015, elaborado em conjunto pelo IBGE e pela EPUSP-Escola Politécnica da Universidade de São Paulo, fruto de mais de duas década de cooperação e de melhorias de versões anteriores. Trata-se de um modelo de ondulação geoidal disponibilizado juntamente com um sistema de interpolação que possibilita aos usuários a obtenção de altitude geoidal em um ponto ou em um conjunto de pontos do território nacional a partir de suas coordenadas. Sua abrangência cobre a área compreendida pelas latitudes $6^{\circ} \mathrm{Ne} 35^{\circ} \mathrm{S}$ e pelas longitudes $75^{\circ} \mathrm{W}$ e $30^{\circ} \mathrm{W}$, referenciadas ao SIRGAS 2000 (IBGE, 2015). Apesar de, claramente, uma análise do exposto em relevantes trabalhos, como IBGE (2015) e Blitzkow et al. (2016), demonstrar que o MAPGEO2015 apresenta melhorias significativas em relação ao MAPGEO2010, adequando-se com precisão centimétrica ao território nacional e em média apresenta diferença nula com valores reais, sua utilização neste trabalho se dá em forma de avaliação e comparação com os MGGs atuais. Isto porque trabalhos anteriores, tais como Melo (2011) e Jamur et al. (2010), demonstram baixa aderência do modelo geoidal nacional em determinadas regiões do país, em detrimento de uso de MGGs. Adicionalmente, a carência de outros trabalhos acadêmicos empregando e avaliando o uso do MAPGEO2015 estimulam seu uso no presente trabalho.

\section{Area de estudo e área-teste}

Conforme já abordado anteriormente, a área de estudo do presente trabalho é a Amazônia Legal Brasileira. Trata-se de uma área de cerca de 5 milhões de quilômetros quadrados - correspondendo a aproximadamente $59 \%$ do território brasileiro-e que, atualmente, corresponde à área dos Estados da Região Norte do Brasil, acrescidos da totalidade do Estado de Mato Grosso e dos municípios do Estado do Maranhão situados a oeste do meridiano $44^{\circ} \mathrm{O}$. Todavia, no que diz respeito à existência de estações que atendam aos pressupostos apresentados até o momento, a área de estudo conta com 154 estações SAT-GPS que possuem conexão com a RAFB - doravante denominadas estações GNSS-RN, distribuídas de forma não-homogênea e perfazendo a área de estudo de referência inicial, conforme Figura 2.

Entretanto, levando-se em consideração a baixa confiabilidade e disponibilidade de dados na área de estudo para fomentar a elaboração de uma metodologia consistente - entenda-se: baixa vinculação à RAFB, encontrando-se em sua maioria em ramais da rede; baixa precisão das altitudes normais-ortométricas; entre outros óbices - julgou-se cabível a adoção de uma área-teste consistente. Esta área-teste foi o estado do Paraná, o qual contava inicialmente com 32 estações GNSS/RN distribuídas de maneira não-uniforme, conforme Figura 3. 


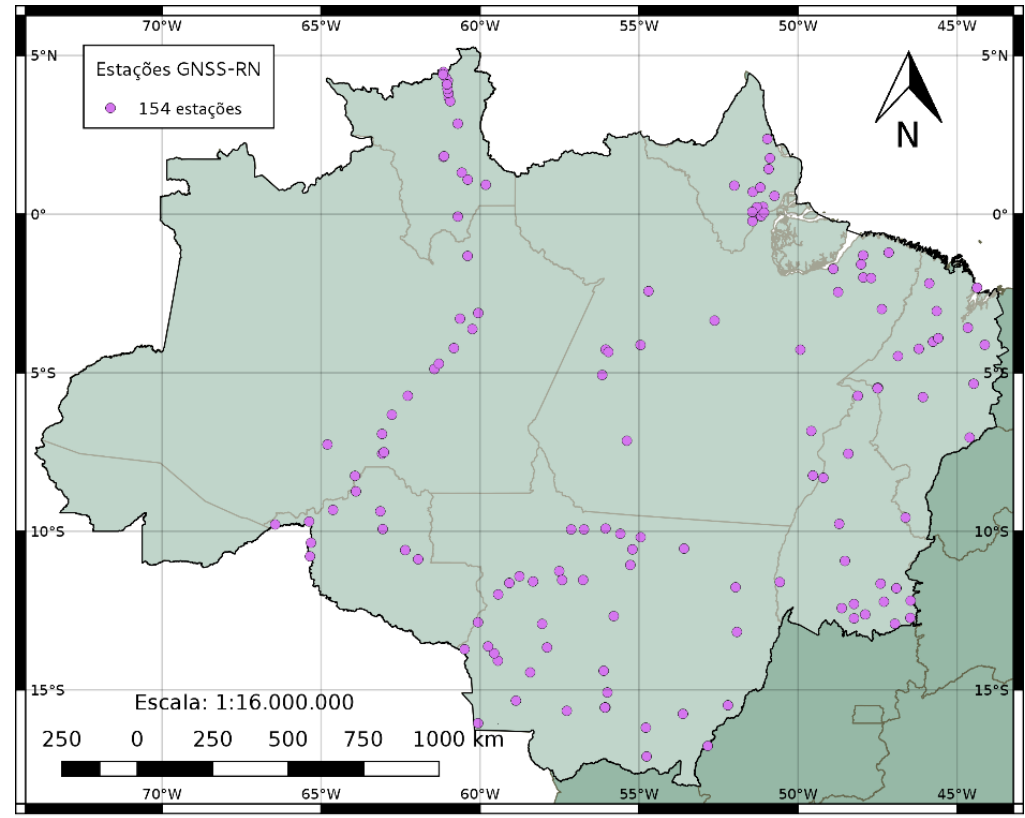

Figura 2. Área de estudo de referência inicial - 154 estações GNSS/RN. Fonte: Nicacio, 2017.

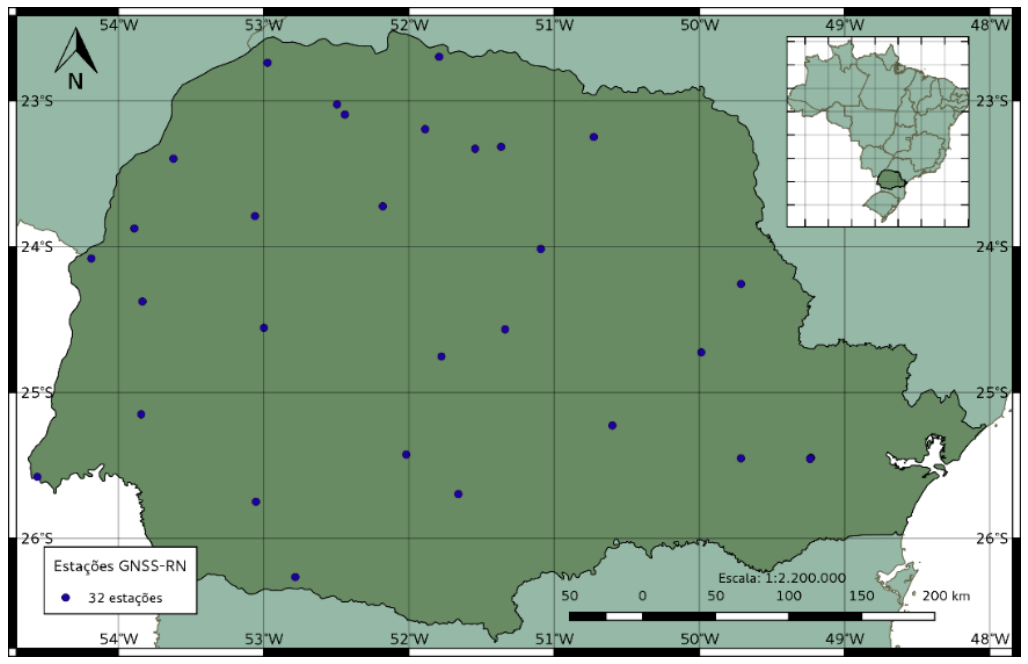

Figura 3. Área-teste de referência inicial - 32 estações GNSS/RN.

Fonte: Nicacio, 2017. 
Como, novamente, a elaboração da metodologia poderia ser prejudicada pela nãohomogeneidade das estações, realizou-se um procedimento para identificação e seleção de estações baseado em coerência entre mútuas distâncias quando comparadas às resoluções espaciais dos MGGs empregados e em análise de suas precisões altimétricas (Nicacio, 2017). Para exemplificar, tome-se como exemplo a obtenção de extratos de MGG com grau máximo de desenvolvimento 720, cujas resoluções seriam, consonante Seeber (2003, p. 469) da ordem de 20.000/720, aproximadamente igual a $27,78 \mathrm{~km}$. Assim, estações GNSS/RN que distem menos de 27,78 km entre si devem ser eliminadas, com exclusão daquelas que possuírem menores precisões.

Destarte, seguindo estes critérios, a quantidade de estações na área-teste de referência final utilizada no presente trabalho foi subdividida da seguinte forma: foram utilizados 22 pontos para modelos desenvolvidos até grau 360, 27 pontos para modelos desenvolvidos até grau 720 e 29 pontos para modelos até grau 2190.

Ainda, para a área de estudo e para a área-teste, foi identificado um conjunto de pontos denominado área desejada, na qual deseja-se realizar a determinação das altitudes normais-ortométricas por meio da estratégia elaborada. A área de estudo desejada é composta por 602 estações SAT-GPS, ao passo que a área-teste desejada é composta por 127 estações da mesma rede.

\section{Estratégia para determinação de altitudes normais-ortométricas}

Para fins de elaboração da estratégia, foram usadas três vertentes distintas de análise para determinação das altitudes normais-ortométricas: a) utilização dos métodos absoluto e relativo para determinação altimétrica; b) utilização de extratos dos MGGs obtidos em diferentes graus de desenvolvimento e do MAPGEO2015; e c) utilização das funcionais altitude geoidal e anomalia de altitude para modelagem de altitude normal-geoidal. Em cada uma delas, elencou-se como mais vantajosa aquela que minimizava o módulo da discrepância entre os valores de altitude normal-ortométrica de referência $\left(H_{r e f}^{N O r t}\right)$, já possuídos pelos pontos, e os valores calculados $\left(H^{\text {NOrt }}{ }_{\text {calc }}\right)$ por meio das vertentes anteriores.

Por exemplo, para fins da comparação entre os métodos absoluto e relativo, considerando-se que as altitudes normais-ortométricas são determinadas pelo método absoluto por intermédio da equação 3 , ao passo que estas são determinadas pelo método relativo por intermédio da equação 4 , considerou-se com melhor desempenho aquela que tornou verdadeira a expressão 6.

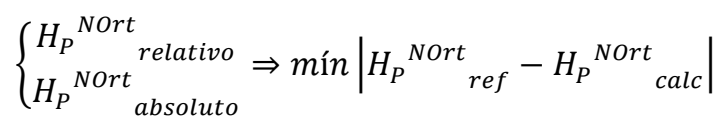

Cada vertente foi analisada individualmente com vistas a escolha das melhores opções para a estratégia consolidada. Adicionalmente, por ocasião do uso do método 
relativo, tal como apontado por Nicacio (2017), dentre os possíveis limitantes para o posicionamento do ponto $P_{0}$, optou-se por adotar aquele posicionado de forma mais centralizada na área do conjunto de pontos considerado, para fins de homogeneização das linhas de base desta determinação.

\section{Principais resultados obtidos}

\section{Resultados para a área-teste}

Através dos estudos realizados para a área-teste, foi possível atestar os principais resultados conforme gráfico apresentado na Figura 4. Neste gráfico, as discrepâncias médias por conjunto de pontos, obtidas por meio do método relativo são representadas por linhas contínuas, ao passo que aquelas obtidas pelo método absoluto são representadas por linhas tracejadas. Na legenda do gráfico, estão destacados no retângulo magenta as representações obtidas pela funcional altitude geoidal e no retângulo ciano aquelas obtidas pela funcional anomalia de altitude.

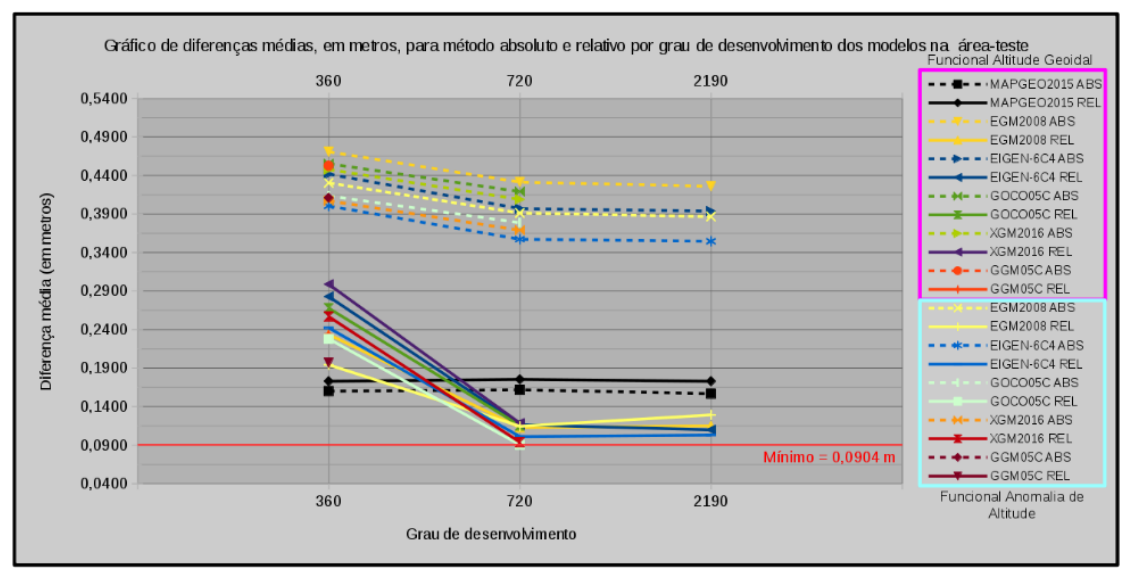

Figura 4. Gráfico de diferenças médias, em metros, para método absoluto e relativo por grau de desenvolvimento dos modelos na área-teste.

Fonte: Nicacio, 2017.

Da figura em questão, pode-se verificar que o desempenho do uso do método relativo é, conforme esperado deveras superior ao uso do método absoluto para os MGGs; Este padrão, no entanto, não é conservado pelo MAPGEO2015. Adicionalmente, não há padrão de comportamento no tocante às diferentes funcionais do geopotencial para modelagem da altitude normal-geoidal, bem como não há escolha crassa para uso de distintos graus de desenvolvimento - exceção feita para extratos desenvolvidos até grau 360, os quais tiveram desempenho aquém do esperado. 
Observe-se, ainda, na Figura 4, a existência de uma configuração que minimizou a discrepância média do conjunto de pontos ao qual ela foi aplicada, da forma da equação 7, na qual há o cálculo da discrepância individual $\theta_{i}$ para cada um dos $i$ pontos de um conjunto de $n$ pontos. Esta configuração foi denominada "configuração ótima" e, para este conjunto de pontos, foi o MGG GOCO05C, desenvolvido até grau 720 e segundo a funcional anomalia de altitude. Com esta configuração, obteve-se discrepância média $\theta_{m}=0,0904 \mathrm{~m}=9,04 \mathrm{~cm}$.

$$
\theta_{i}=\left|H_{P}{ }_{\text {ref }}^{\text {Nort }}-H_{P}{ }_{\text {calc }}^{\text {NOrt }}\right| \Rightarrow \theta_{m}=\frac{1}{n} \sum_{i=1}^{n} \theta_{i}
$$

Para a área-teste pode-se, por fim, elaborar a Tabela 2, a qual demonstra os ganhos impostos pela utilização da metodologia alternativa, que contempla o uso dos MGGs em sua configuração ótima associados ao método relativo, em comparação a uma metodologia denominada tradicional, que contempla o uso do MAPGEO2015 associado ao método absoluto. Estas estimativas finais foram realizadas após uma fase de identificação visual e eliminação de outliers, ou seja, pontos cuja modelagem não foi bem executada segundo nenhuma das configurações utilizadas.

Tabela 2

Comparativo entre metodologia proposta e metodologia tradicional na área-teste

\begin{tabular}{lllllll}
\hline & \multicolumn{3}{l}{ Metodologia alternativa } & \multicolumn{4}{l}{ Metodologia tradicional } \\
\cline { 2 - 6 } & $\theta(\mathrm{m})$ & $\sigma_{\theta}{ }^{2}\left(\mathrm{~m}^{2}\right)$ & $\sigma_{\theta}(\mathrm{m})$ & $\theta(\mathrm{m})$ & $\sigma_{\theta}{ }^{2}\left(\mathrm{~m}^{2}\right)$ & $\sigma_{\theta}(\mathrm{m})$ \\
\hline Máxima & 0,1487 & & & 0,5304 & & \\
Média & 0,0621 & 0,0015 & 0,0393 & 0,1678 & 0,0118 & 0,1085 \\
Mínima & 0,0005 & & & 0,0022 & & \\
\hline
\end{tabular}

Fonte: Nicacio (2017).

Sendo assim, os ganhos da aplicação da metodologia ora proposta foram reais e de grande significância para a aplicação da estratégia alternativa, sendo em média superiores a 10 centímetros para a área-teste. Estes resultados apontaram no sentido de refinamento da metodologia utilizada, elaboração de uma metodologia consolidada e sua precípua aplicação na área de estudo.

\section{Estratégia alternativa consolidada}

Da análise da Figura 4, bem como de outras análises realizadas, verificou-se os tópicos apresentados a seguir. Estes foram considerados por ocasião da elaboração da estratégia alternativa consolidada, aplicável à área de estudo e extensível a demais áreas onde se almeje realizar abordagem similar. 
- O uso do método relativo é comprovadamente mais adequado que o método absoluto para manipulação dos MGGs; estes devem, portanto, incorporar a estratégia definitiva;

- A partir de todo o conjunto de pontos disponíveis em uma determinada área, deve ser mantida a seleção e priorização de pontos para homogeneização de dados, segundo diferentes graus de desenvolvimento dos extratos dos MGGs e suas resoluções espaciais;

- Em virtude de seu desempenho aquém do esperado e de sua inadequação para uso segundo os pressupostos do método relativo, a funcional altitude geoidal obtida por meio do modelo geoidal nacional MAPGEO2015 deve ser descartada da estratégia consolidada;

- Quando da utilização do método relativo, deverá ser adotado o ponto $P_{0}$ mais próximo do centro da área estudada, como aproximação primária para seu baricentro, haja visto que esta consideração demonstra-se suficientemente eficaz para o princípio que ora se propõe e que outras configurações testadas não apresentaram ganho significativo;

- Quando da obtenção de extratos dos MGGs serão utilizados os modelos EGM2008, EIGEN-6C4, GOCO05C e XGM2016, segundo graus de desenvolvimento 720 e 2190 - exceção feita ao MGG XGM2016, para o qual será utilizado seu grau máximo 719 - em virtude destes originarem funcionais do geopotencial que melhor representam a altitude normal-geoidal;

- Ainda quando da obtenção de extratos dos MGGs, serão consideradas as funcionais anomalia de altitude e altitude geoidal, vislumbrando a modelagem da altitude normal-geoidal, uma vez que não houve comportamento padronizado para os resultados neste aspecto;

- A escolha da configuração ótima se dará para o MGG, o grau de desenvolvimento e a funcional que minimizarem o fator $\theta_{m}$ para o conjunto de pontos estudado;

- Após a determinação das altitudes normais-ortométricas em área desejada, deve ser realizada uma validação dos resultados em uma área desejada controlável, com precípua eliminação de outliers;

\section{Avanços na determinação de altitudes normais-ortométricas na área de estudo}

Analogamente ao desenvolvido na área-teste, realizou-se a busca por uma configuração ótima na área de estudo, a qual foi identificada como o GGM XGM2016, desenvolvido até grau 719 e segundo a funcional anomalia de altitude; segundo esta configuração, obteve-se discrepância média $\theta_{m}=0,2160 \mathrm{~m}=21,60 \mathrm{~cm}$. A Figura 5 apresenta gráfico similar àquele disposto na Figura 4 , para esta área.

Para a área de estudo pode-se, também, elaborar a Tabela 3, a qual demonstra os ganhos impostos pela utilização da metodologia alternativa em comparação a uma metodologia tradicional, analogamente ao realizado na Tabela 2. Novamente, estas estimativas finais foram realizadas após uma fase de identificação e eliminação de outliers, baseada em um critério de até um desvio-padrão de diferença em relação à 
média do conjunto de pontos. Das 136 estações GNSS/RN utilizadas para este fim, 22 foram eliminadas com este critério, indicando possíveis problemas em suas determinações altimétricas originais.

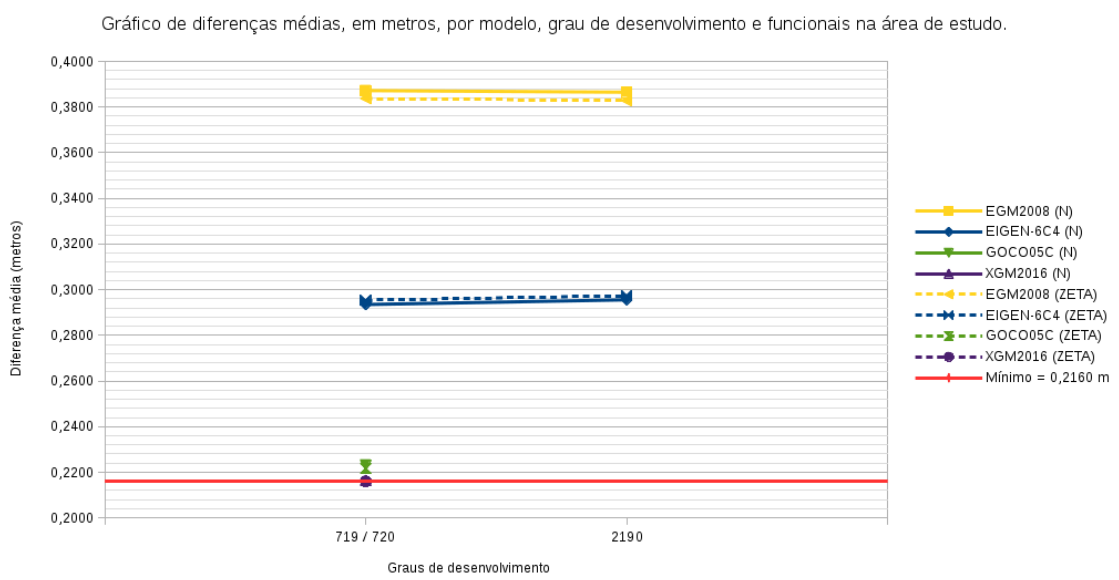

Figura 5. Gráfico de diferenças médias, em metros, para método absoluto e relativo por grau de desenvolvimento dos modelos na área de estudo.

Fonte: Nicacio, 2017.

Tabela 3

Comparativo entre metodologia proposta e metodologia tradicional na área de estudo

\begin{tabular}{lllllll}
\hline & \multicolumn{3}{l}{ Metodologia alternativa } & \multicolumn{3}{l}{ Metodologia tradicional } \\
\cline { 2 - 6 } & $\theta(\mathrm{m})$ & $\sigma_{\theta}{ }^{2}\left(\mathrm{~m}^{2}\right)$ & $\sigma_{\theta}(\mathrm{m})$ & $\theta(\mathrm{m})$ & $\sigma_{\theta}{ }^{2}\left(\mathrm{~m}^{2}\right)$ & $\sigma_{\theta}(\mathrm{m})$ \\
\hline Máxima & 0,3665 & & & 0,9029 & & \\
Média & 0,1387 & 0,0112 & 0,1057 & 0,3628 & 0,0468 & 0,2164 \\
Mínima & 0,0006 & & & 0,0244 & & \\
\hline
\end{tabular}

Fonte: Nicacio (2017).

Sendo assim, novamente atesta-se que os ganhos da aplicação da metodologia ora proposta são reais e de grande significância para a aplicação da estratégia alternativa. Estes resultados confirmam a superioridade da metodologia e da estratégia consolidada utilizadas, proporcionando ganhos superiores a 20 centímetros para determinações altimétricas na complexa Região Amazônica. 


\section{Estimativa do off-set entre DVB-I e DVB-S}

Ao final dos trabalhos, verificou-se ainda a possibilidade de realizar uma nova estimativa da separação vertical (off-set) entre os data verticais brasileiros - DVB-I, em Imbituta-SC, e DVB-S, em Santana-AP - com base no uso da metodologia proposta. Aprofundamentos sobre esta abordagem podem ser encontrados no trabalho original; entretanto, os resultados encontrados mostram-se deveras consistentes com aqueles já obtidos anteriormente, conforme apontado na tabela 4. As principais discrepâncias são devidas às imprecisões dos dados de entrada para a metodologia, bem como a diferentes abordagens para solução do problema.

\section{Tabela 4}

Atualização de autores e valores determinados de off-set entre data verticais

\begin{tabular}{cc}
\hline Autor & Off-set $(\mathrm{m})$ \\
\hline Nicacio (2017) & $1,320 \pm 0,07 \mathrm{~m}$ \\
De freitas et al. (2016) & $1,416 \pm 0,12 \mathrm{~m}$ \\
Moreira (2015) & $1,300 \pm 0,11 \mathrm{~m}$ \\
Castro e De Freitas (2014) & 1,301 a $1,420 \mathrm{~m}$ \\
Castro (2011) & $1,320 \mathrm{~m}$ ou $1,430 \mathrm{~m}$ \\
\hline
\end{tabular}

Fonte: Nicacio (2017).

\section{Conclusões e Contribuições do Trabalho}

Foi desenvolvida uma ferramenta, em duas versões conforme necessidade, para rápida obtenção de extratos de MGGs ponto-a-ponto com acesso direto ao serviço web do ICGEM: o SPGG (Nicacio, 2016). Com seu uso, foram obtidos dados necessários para este e para outros trabalhos, de tal maneira que o usuário regular não necessite realizar manipulações matemáticas complexas com os coeficientes do desenvolvimento dos modelos em harmônicos esféricos, tampouco dependa da interação repetitiva com o referido serviço. Esta ferramenta encontra-se disponível para download no endereço da referência e pode ser livremente utilizada pela comunidade, principalmente para o público que necessita trabalhar com extratos de modelos globais e não deseja realizar uma manipulação direta de seus coeficientes.

Foi analisado o desempenho do uso do método relativo ante ao método absoluto para predições altimétricas baseadas no uso de MGGs e de altitudes elipsoidais. Esta pode ser considerada a principal contribuição do presente trabalho, tendo em vista que os resultados das análises a ela referentes indicam que a maneira ideal de lidar com os MGGs e suas funcionais é, de fato, com o uso do método relativo, tomadas 
precauções quanto ao pleno alinhamento com conceitos físicos atinentes. Foi analisado o desempenho de expoentes MGGs combinados da atualidade em porções específicas do território nacional - a saber, estado do Paraná e Amazônia Legal Brasileira - quanto à adequação para modelagem da altitude normal-geoidal. Esta contribuição fomentou ainda a confecção de artigo acadêmico com abrangência do território nacional em completude.

Foram desenvolvidas especificações e orientações quanto à metodologia alternativa para predição de altitudes normais-ortométricas com base em MGGs e suas funcionais, observações GNSS e uso do método relativo, com vistas a densificar a informação altimétrica na região de carência na Amazônia Legal Brasileira. Estas orientações podem ser expandidas para emprego em todo o território nacional conforme necessidade, desde que conservadas as precauções imperativas descritas ao longo do trabalho. Os estudos demonstraram uma necessidade de revisão em normativas existentes que apontam para o uso do método absoluto como alternativa para o nivelamento geométrico quando da predição de altitudes normais-ortométricas, como aquelas preconizadas por Aneel (2009, p. 23) para pequenas centrais hidrelétricas, e a implementação de normatização para uso dos MGGs.

Por fim, proveu-se uma nova estimativa para o afastamento entre os data verticais brasileiros, baseado no emprego da metodologia desenvolvida. Seu resultado coerente com as demais estimativas existentes confirmam a correção da metodologia desenvolvida e dos processos empregados, somando-se a estes resultados anteriores como possíveis valores para o off-set em questão.

\section{Bibliografia}

Abd-Elmotaal, H.A. (2015). "Validation of GOCE Models in South Africa", Newton's Bulletin, Assessment of GOCE Geopotential Models, vol. 5, pp. 149-162.

Al-Ghamdi, K.A. and Dawod, G.M. (2014). "Accuracy Assessment of Global Geopotential Models for GIS and Geomatics Applications in Makkah Metropolitan Area", in The 9th National GIS Symposium in Saudi Arabia, Saudi Arabia.

Aneel (2009). Diretrizes para elaboração de serviços de Cartografia e Topografia, relativos a estudos e projetos de aproveitamento hidrelétricos, Superintendência de Gestão e Estudos Hidroenergéticos (SGH).

Arana, J.M. (2000). “O uso do GPS na elaboração da carta geoidal”, Tese Doutorado em Ciências Geodésicas, Programa de Pós-Graduação em Ciências Geodésicas, Setor de Ciências da Terra, Universidade Federal do Paraná, Curitiba.

Barthelmes, F. and Köhler, W. (2016). "International Centre for Global Earth Models (ICGEM)", in Drewes H.; Kuglitsch, F. and Adam, J. (eds.), The Geodesists Handbook 2016. Journal of Geodesy, vol. 90, cap. 10, pp. 907-1205. 
Blitzkow, D.; Matos, A.C.O.C.; Machado; W.C.; Nunes, M.A.; Lengruber, N.V.; Xavier, E.M.L. and Fortes, L.P.S. (2016). "MAPGEO2015: O novo modelo de ondulação geoidal do Brasil", Revista Brasileira de Cartografia, vol. 68, no. 10, pp. 1873-1884.

Castro, H.M. (2011). "Proposta de Alternativas para conexão dos Data Verticais Brasileiros de Imbituba e Santana", Dissertação Mestrado em Ciências Geodésicas, Programa de Pós-Graduação em Ciências Geodésicas, Setor de Ciências da Terra, Universidade Federal do Paraná, Curitiba.

Castro, H.D.M. and De Freitas, S.R.C. (2014). "Strategies for Connecting Imbituba and Santana Brazilian Datums Based on Satellite Gravimetry and Residual Terrain Model", in Rizos, P.W.C. (ed.), Earth on the Edge: Science for a Sustainable Planet, Berlin, Springer, vol. 139, pp. 543-550.

De Freitas, S.R.C.; Santacruz Jaramillo, A.G.; Luz, R.T.; Ferreira, V.G.; Castro, H. D.M. and Sánchez, J.L.C. (2016). "Connection of the two segments of the Brazilian Vertical Reference Network", in 1st Joint Comission 2 and International Symposium on Gravity, Geoid and Height Systems 2016, Thessaloniki, Grécia.

Drewes, H. (2006). "The changing objectives in geodetic research", Zeitschrift für Geodäsie, Geo-Information und Landmanagement, vol. 131, no. 5, pp. 1-7.

Featherstone, W.E. (2002). "Expected contributions of dedicated satellite gravity field missions to regional geoid determination with some examples from Australia", Journal of Geospatial Engineering, vol. 4, pp. 2-19.

Ferreira, V.G.; Zhang, Y. and De Freitas, S.R.C. (2013). "Validation of GOCE gravity field models using GPS-leveling data and EGM08: a case study in Brazil", Journal of Geodetic Science, vol. 3, no. 3, pp. 209-218.

Gemael, C. (2012). Introdução à Geodésia Física, Curitiba, Editora UFPR.

Godah, W.; Krynski, J. and Szelachowska, M. (2015). "On the accuracy assessment of the consecutive releases of GOCE-based GGMs over the area of Poland", Newton's Bulletin. Assessment of GOCE Geopotential Models, vol. 5.

Hofmann-Wellenhof, B. and Moritz, H. (2006). Physical Geodesy, 2nd. ed. Graz, Austria, Spring-Verlag Wien.

IAG (2015). Resolution (No. 1) for the definition and realization of an International Height Reference System (IHRS), München, Alemanha.

IBGE (2011). Ajustamento Simultâneo da Rede Altimétrica de Alta Precisão do Sistema Geodésico Brasileiro, Rio de Janeiro.

- (2015). Relatório de Desenvolvimento do MAPGEO2015, Rio de Janeiro.

(acesso em 2017). SGB - Sistema Geodésico Brasileiro. Disponível em: $<$ http://www.ibge.gov.br/home/geociencias/geodesia/default_sgb_int.shtm>.

INDE (acesso em 2016). Visualizador da INDE. Disponível em: <http://www.visualizador.inde.gov.br/>. 
Jamur, K.P.; Melo, L. and De Freitas, S.R.C. (2010). "Avaliação dos Modelos do Campo da Gravidade Terrestre advindos da Missão GOCE - Gravity Field and Steady-State Ocean Circulation Explorer", in Reunión Anual SIRGAS 2010, Lima, Peru. Boletín Informativo no. 15 SIRGAS Reporte 2009-2010.

Matos, A.C.O.C.; Guimarães, G.N.; Lobianco, M.C.B. and Campos, I.O. "Evaluation of Recent GOCE Geopotential Models in South America", Newton's Bulletin, Assessment of GOCE Geopotential Models, vol. 5, pp. 83-104.

Melo, L.F.S. (2011). "Avaliação de modelos globais do geopotencial para os estados do Maranhão e Piauí", dissertação Mestrado em Ciências Geodésicas, Programa de Pós-Graduação em Ciências Geodésicas, Setor de Ciências da Terra, Universidade Federal do Paraná, Curitiba.

Moreira, R.M. (2015). "Conexão de Sistemas Verticais de Referência Locais ao Sistema Geodésico Brasileiro com base em um Sistema Vertical de Referência Global", Tese Doutorado em Ciências Geodésicas, Programa de Pós-Graduação em Ciência Geodésicas, Setor de Ciências da Terra, Universidade Federal do Paraná, Curitiba.

Nicacio, E.L. (acesso em 2016). "SPGG - Single Point GEM Generator”. Disponível em: $<$ http://euriconicacio.github.io/spgg/>.

(2017). "Alternativa para determinação de altitudes normais-ortométricas na Amazônia Legal Brasileira". Dissertação Mestrado em Ciências Geodésicas, Programa de Pós-Graduação em Ciências Geodésicas, Setor de Ciências da Terra, Universidade Federal do Paraná, Curitiba.

Nicacio, E.L.; Dalazoana, R. (2017). "Passado e Presente dos Modelos Globais do Geopotencial: Uma Abordagem Conceitual Sobre sua Evolução", Revista Eletrônica Multidisciplinar FACEAR, vol. 2, pp. 1-15.

Pavlis, N.K. et al. (2012). "The development and evaluation of the Earth Gravitational Model 2008 (EGM2008)", Journal of Geophysical Research, vol. 117, no. B04406, pp. 1-38.

Sánchez, L. (2016). "Sistemas de referencia vertical", in Taller SIRGAS en Sistemas Verticales de Referencia 2016, Quito, Ecuador.

Santacruz Jaramillo, A.G. et al. (2016). "Estrategias para la conexión de redes verticales heterogéneas con base en el PVCG en la forma fija", in Symposium SIRGAS 2016, Quito, Ecuador.

Seeber, G. (2003). “Satellite Geodesy: foundations, methods and applications", Berlin-New York, Walter de Gruyter.

Torge, W. and Müller, J. (2012). Physical Geodesy, 4th. ed. Berlin-Boston, Walter de Gruyter, 2012.

Voigt, C. and Denker, H. (2015). "Validation of GOCE Gravity Field Models in Germany", Newton's Bulletin, Assessment of GOCE Geopotential Models, vol. 5, pp. $37-48$. 